\title{
B decays to open charm
}

\author{
Susan Haines*t \\ University of Cambridge \\ E-mail: haines@hep.phy.cam.ac.uk
}

Studies of $B$ meson decays to states involving open charm mesons in data recorded by the LHCb experiment have resulted in first observations of several new decay modes, including $B_{s}^{0} \rightarrow D_{s}^{* \mp} K^{ \pm}, B_{s}^{0} \rightarrow \bar{D}^{0} K_{S}^{0}$ and $B^{+} \rightarrow D^{+} K^{+} \pi^{-}$decays. An upper limit has been placed on the branching fraction of $B_{s}^{0} \rightarrow \bar{D}^{0} f_{0}(980)$ decays. Measurements of other branching fractions, such as those of $B_{s}^{0} \rightarrow D_{s}^{(*)+} D_{s}^{(*)-}$ decays, are the most precise to date. Additionally, amplitude analyses of $B^{0} \rightarrow \bar{D}^{0} \pi^{+} \pi^{-}$and $B^{0} \rightarrow \bar{D}^{0} K^{+} \pi^{-}$decays have been performed, alongside the first $C P$ violation analysis using the Dalitz plot of $B^{0} \rightarrow D K^{+} \pi^{-}$decays.

16th International Conference on B-Physics at Frontier Machines

2-6 May 2016

Marseille, France

*Speaker.

${ }^{\dagger}$ On behalf of the LHCb collaboration. 


\section{Measurement of the $B_{s}^{0} \rightarrow D_{s}^{(*)+} D_{s}^{(*)-}$ branching fractions}

The branching fractions of $B_{s}^{0} \rightarrow D_{s}^{(*)+} D_{s}^{(*)-}$ decays ${ }^{1}$ provide an important contribution to the inclusive branching fraction for $b \rightarrow c \bar{c} s$ quark transitions. Precise measurements of the branching fractions are therefore vital, allowing model-independent searches for physics beyond the Standard Model to be performed [1]. These measurements can also aid the understanding of hadronisation effects in $B_{s}^{0}$ meson decays via the $b \rightarrow c \bar{c} s$ transition. Measurements of the branching fractions have been made using data recorded at $\mathrm{LHCb}$ [2], giving the most precise results to date,

$$
\begin{aligned}
\mathscr{B}\left(B_{s}^{0} \rightarrow D_{s}^{(*)+} D_{s}^{(*)-}\right) & =(3.05 \pm 0.10 \pm 0.20 \pm 0.34) \%, \\
\mathscr{B}\left(B_{s}^{0} \rightarrow D_{s}^{* \pm} D_{s}^{\mp}\right) & =(1.35 \pm 0.06 \pm 0.09 \pm 0.15) \% \text { and } \\
\mathscr{B}\left(B_{s}^{0} \rightarrow D_{s}^{*+} D_{s}^{*-}\right) & =(1.27 \pm 0.08 \pm 0.10 \pm 0.14) \%,
\end{aligned}
$$

where the first uncertainties are statistical, the second are systematic and the third arise from the uncertainty on the branching fraction of the normalisation decay $B^{0} \rightarrow D_{s}^{+} D^{-}$.

\section{First observation and measurement of the branching fraction for the decay}

$B_{s}^{0} \rightarrow D_{s}^{* \mp} K^{ \pm}$

The decay $B_{s}^{0} \rightarrow D_{s}^{* \mp} K^{ \pm}$has been observed for the first time, using data collected at the LHCb experiment [3]. Its branching fraction relative to that for $B_{s}^{0} \rightarrow D_{s}^{*-} \pi^{+}$decays has been measured to be $\mathscr{B}\left(B_{s}^{0} \rightarrow D_{s}^{* \mp} K^{ \pm}\right) / \mathscr{B}\left(B_{s}^{0} \rightarrow D_{s}^{*-} \pi^{+}\right)=0.068 \pm 0.005_{-0.002}^{+0.003}$, where the first uncertainty is statistical and the second is systematic. The measured value of the branching fraction ratio is consistent with theoretical predictions [4]. In future, $B_{s}^{0} \rightarrow D_{s}^{* \mp} K^{ \pm}$decays could be used to measure the weak phase $\gamma$.

\section{Observation of $B_{s}^{0} \rightarrow \bar{D}^{0} K_{S}^{0}$ and evidence for $B_{s}^{0} \rightarrow \bar{D}^{* 0} K_{S}^{0}$ decays}

Using data recorded at $\mathrm{LHCb}$, the decay $B_{s}^{0} \rightarrow \bar{D}^{0} K_{S}^{0}$ has been observed for the first time, and evidence of the decay $B_{s}^{0} \rightarrow \bar{D}^{* 0} K_{S}^{0}$ has been found [5]. The measured branching fractions are

$$
\begin{aligned}
\mathscr{B}\left(B_{s}^{0} \rightarrow \bar{D}^{0} \bar{K}^{0}\right) & =(4.3 \pm 0.5 \pm 0.3 \pm 0.3 \pm 0.6) \times 10^{-4} \text { and } \\
\mathscr{B}\left(B_{s}^{0} \rightarrow \bar{D}^{* 0} \bar{K}^{0}\right) & =(2.8 \pm 1.0 \pm 0.3 \pm 0.2 \pm 0.4) \times 10^{-4},
\end{aligned}
$$

where the first uncertainties are statistical, the second are systematic, the third are due to the ratio of fragmentation fractions $\left(f_{s} / f_{d}\right)$ and the fourth arise from the uncertainty on the branching fraction of the normalisation decay $B^{0} \rightarrow \bar{D}^{0} K_{S}^{0}$. These values are consistent with theoretical predictions [6]. The normalisation decay is itself of interest because it could be used to measure $\gamma$ [7], while $B_{s}^{0} \rightarrow \bar{D}^{(*) 0} K_{S}^{0}$ decays offer sensitivity to the $B_{s}^{0}$ meson mixing phase $\phi_{s}$ [8].

\section{Search for the decay $B_{s}^{0} \rightarrow \bar{D}^{0} f_{0}(980)$}

A search for $B_{s}^{0} \rightarrow \bar{D}^{0} f_{0}(980)$ decays has been performed using LHCb data [9]. Measurements of the relative production of scalar mesons in $B_{(s)}^{0}$ meson decays provide insight into their substructure [10]; $\mathscr{B}\left(B^{0} \rightarrow \bar{D}^{0} f_{0}(980)\right)$ has already been measured [11, 12]. Additionally, $B_{s}^{0} \rightarrow \bar{D}^{0} f_{0}(980)$ decays could be used to measure $\gamma$, under the assumption that the $f_{0}(980)$ meson has a predominant $s \bar{s}$ component [7,13]. No significant signal is observed in the data, so upper limits on the branching fraction of $\mathscr{B}\left(B_{s}^{0} \rightarrow \bar{D}^{0} f_{0}(980)\right)<3.1(3.4) \times 10^{-6}$ are set at $90 \%$ (95\%) confidence level.

\footnotetext{
${ }^{1}$ The inclusion of charge conjugate processes is implied throughout this contribution.
} 


\section{First observation of the rare $B^{+} \rightarrow D^{+} K^{+} \pi^{-}$decay}

Using data collected at $\mathrm{LHCb}$, the decay $B^{+} \rightarrow D^{+} K^{+} \pi^{-}$has been observed for the first time [14] with a branching fraction of $\mathscr{B}\left(B^{+} \rightarrow D^{+} K^{+} \pi^{-}\right)=(5.31 \pm 0.90 \pm 0.48 \pm 0.35) \times 10^{-6}$, where the uncertainties are statistical, systematic and due to the uncertainty on the branching fraction of the normalisation decay $B^{+} \rightarrow D^{-} K^{+} \pi^{+}$[15], respectively. Figure 1 shows the corresponding weighted invariant mass distribution for the candidate decays. The Dalitz plot, also shown in Figure 1, appears to be dominated by broad structures; angular distributions are used to search for quasi-two-body contributions from $B^{+} \rightarrow D_{2}^{*}(2460)^{0} K^{+}$and $B^{+} \rightarrow D^{+} K^{*}(892)^{0}$ decays, but no significant signals are seen and upper limits are therefore set on their branching fractions. In future, decays of the type $B^{+} \rightarrow D^{* *} K^{+}$, where $D^{* *}$ represents an excited state such as $D_{2}^{*}(2460)^{0}$ that can decay to both $D^{ \pm} \pi^{\mp}$ and $D \pi^{0}$, could be used to measure $\gamma[16]^{2}$.
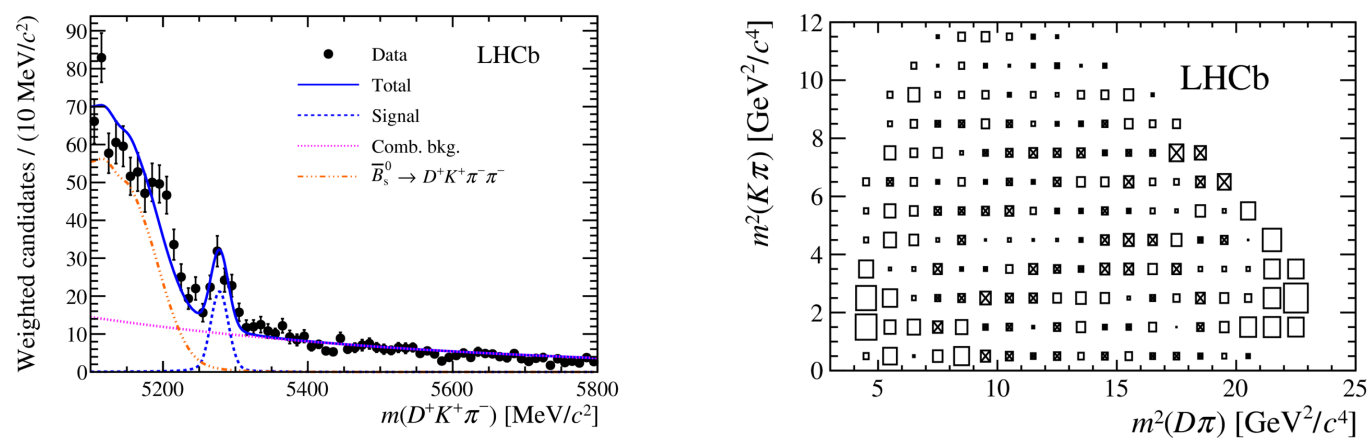

Figure 1: Weighted invariant mass distribution (left) and background-subtracted Dalitz plot distribution (right) of candidate $B^{+} \rightarrow D^{+} K^{+} \pi^{-}$decays [14]. Areas of boxes in the Dalitz plot are proportional to signal yields.

\section{Dalitz plot analysis of $B^{0} \rightarrow \bar{D}^{0} \pi^{+} \pi^{-}$decays}

An amplitude analysis of the decay $B^{0} \rightarrow \bar{D}^{0} \pi^{+} \pi^{-}$has been performed using LHCb data [12]. In the phase-space region $m\left(\bar{D}^{0} \pi^{ \pm}\right)>2.1 \mathrm{GeV} / c^{2}$, the branching fraction of the decay is measured to be $\mathscr{B}\left(B^{0} \rightarrow \bar{D}^{0} \pi^{+} \pi^{-}\right)=(8.46 \pm 0.14 \pm 0.29 \pm 0.40) \times 10^{-4}$, where the first uncertainty is statistical, the second is systematic and the third arises from the uncertainty on the branching fraction of the normalisation decay $B^{0} \rightarrow D^{*}(2010)^{-} \pi^{+}$. The Dalitz plot, shown in Figure 2, is analysed using a model with four components for $\bar{D}^{0} \pi^{-}$resonances, four P-wave $\pi^{+} \pi^{-}$resonances, one Dwave $\pi^{+} \pi^{-}$resonance and two alternative model contributions for the $\pi^{+} \pi^{-}$S-wave components. The complex coefficients and fit fractions for the components of the model are determined from the data. The presence of a resonant structure is confirmed at $m\left(\bar{D}^{0} \pi^{-}\right) \approx 2.8 \mathrm{GeV} / c^{2}$, with its spinparity of $J^{P}=3^{-}$established for the first time; the branching fractions, masses and widths of this resonant structure and the $D_{0}^{*}(2400)^{-}$and $D_{2}^{*}(2460)^{-}$resonances are determined. The branching fractions of $B^{0} \rightarrow \bar{D}^{0} h^{0}\left(\rightarrow \pi^{+} \pi^{-}\right)$decays are also measured, many with the highest precision to date, and several decays are observed for the first time. As well as studying the rich resonant structure of the $B^{0} \rightarrow \bar{D}^{0} \pi^{+} \pi^{-}$decay, the amplitude analysis is an initial step towards a measurement of the CKM angle $\beta$ [17]. Furthermore, $B^{0} \rightarrow \bar{D}^{0} \pi^{+} \pi^{-}$decays offer sensitivity to physics beyond the Standard Model [18].

\footnotetext{
${ }^{2}$ Here, and in Section 8,D denotes a superposition of the $D^{0}$ and $\bar{D}^{0}$ states decaying to the same final state (in Section 5, specifically a $C P$ eigenstate).
} 


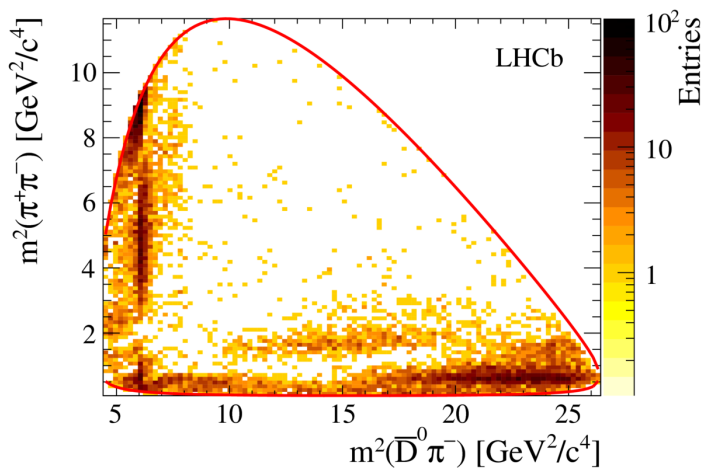

Figure 2: Dalitz plot distribution of candidate $B^{0} \rightarrow \bar{D}^{0} \pi^{+} \pi^{-}$decays [12]. The red line indicates the kinematic boundary of the Dalitz plot.

\section{Amplitude analysis of $B^{0} \rightarrow \bar{D}^{0} K^{+} \pi^{-}$decays}

An amplitude analysis of $B^{0} \rightarrow \bar{D}^{0} K^{+} \pi^{-}$decays in LHCb data has also been performed [19]. The Dalitz plot is analysed using an amplitude model with components for $K^{*}(892)^{0}, K^{*}(1410)^{0}$, $K_{2}^{*}(1430)^{0}$ and $D_{2}^{*}(2460)^{-}$resonances, a $K \pi \mathrm{S}$-wave component, and $D \pi \mathrm{S}$ - and P-wave components. The masses and widths of the $D_{0}^{*}(2400)^{-}$and $D_{2}^{*}(2460)^{-}$resonances are measured and are found to be consistent with those determined in the analysis of $B^{0} \rightarrow \bar{D}^{0} \pi^{+} \pi^{-}$decays described in Section 6; the complex amplitudes and fit fractions for all amplitude model components are also determined.

\section{Constraints on the unitarity triangle angle $\gamma$ from Dalitz plot analysis of $B^{0} \rightarrow D K^{+} \pi^{-}$decays}

Using an amplitude model derived from the results of Ref. [19], described in Section 7, the first $C P$ violation analysis using the Dalitz plot of $B^{0} \rightarrow D\left(\rightarrow K^{+} \pi^{-}, K^{+} K^{-}, \pi^{+} \pi^{-}\right) K^{+} \pi^{-}$decays to measure $\gamma$ [20] has been performed with data recorded at $\mathrm{LHCb}$ [21]. Due to the ability to exploit interference between different contributions to the decay, this method obtains additional sensitivity compared to the quasi-two-body analysis, where only the $K^{*}(892)^{0}$ resonance region of the Dalitz plot is used. No significant $C P$ violation effect is observed; constraints are placed on $\gamma$ using the $B^{0} \rightarrow D K^{*}(892)^{0}$ contribution to the decay, with no value of $\gamma$ excluded at $95 \%$ confidence level, as shown in Figure 3. Hadronic parameters required to determine $\gamma$ from quasi-two-body analyses of $B^{0} \rightarrow D K^{*}(892)^{0}$ decays are also measured. These measurements provide important input to the determination of $\gamma$ from a combination of $B \rightarrow D K$ analyses using LHCb data [22].

\section{Conclusions and prospects}

Many recent studies of $B$ meson decays to open charm have been performed using data recorded by the LHCb experiment, resulting in first observations of several decay modes and world-best measurements of others. There are also excellent prospects for the future use of some of these decays for $C P$ violation measurements, including measurements of the CKM angles $\gamma$ and $\beta$. Studies of further decay modes and analysis updates to include new data collected at the LHCb experiment will provide additional and improved measurements in the near future. 


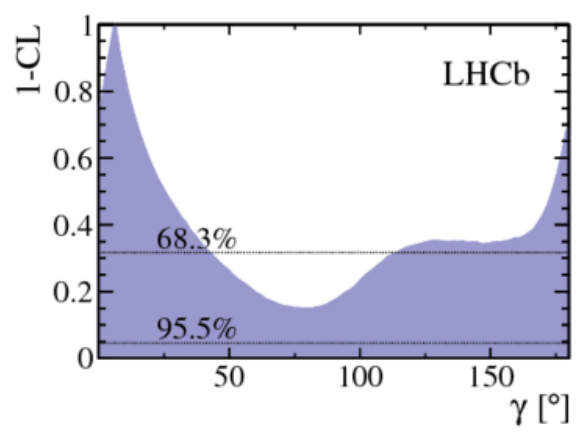

Figure 3: Constraints on $\gamma$ from the $B^{0} \rightarrow D K^{*}(892)^{0}$ contribution to $B^{0} \rightarrow D K^{+} \pi^{-}$decays [21].

\section{Acknowledgement}

The speaker expresses her gratitude for the generous support of the Leverhulme Trust in funding her participation in the conference.

\section{References}

[1] F. Krinner, A. Lenz, T. Rauh, The inclusive decay $b \rightarrow c \bar{c} s$ revisited, Nucl. Phys. B 876 (2013) 31 [arXiv:1305.5390]

[2] LHCb collaboration, Measurement of the $B_{s}^{0} \rightarrow D_{s}^{(*)+} D_{s}^{(*)-}$ branching fractions, Phys. Rev. D 93 (2016) 092008 [arXiv: 1602.07543 ]

[3] LHCb collaboration, First observation and measurement of the branching fraction for the decay $B_{s}^{0} \rightarrow D_{s}^{* \mp} K^{ \pm}$, JHEP 06 (2015) 130 [arXiv: 1503.09086 ]

[4] K. De Bruyn et al., Exploring $B_{s} \rightarrow D_{s}^{(*) \pm} K^{\mp}$ decays in the presence of a sizable width difference $\Delta \Gamma_{s}$, Nucl. Phys. B 868 (2013) 351 [arXiv: 1208 . 6463]

[5] LHCb collaboration, Observation of $B_{s}^{0} \rightarrow \bar{D}^{0} K_{S}^{0}$ and evidence for $B_{s}^{0} \rightarrow \bar{D}^{* 0} K_{S}^{0}$ decays, Phys. Rev. Lett. 116 (2016) 161802 [arXiv: 1603.02408 ]

[6] P. Colangelo, R. Ferrandes, Model independent analysis of a class of $\bar{B}_{s}^{0}$ decay modes, Phys. Lett. $B$ 627 (2005) 77 [arXiv: hep-ph $/ 0508033$ ]; C.-K. Chua, W.-S. Hou, Rescattering effects in $\bar{B}_{u, d, s} \rightarrow D P, \bar{D} P$ decays, Phys. Rev. D 77 (2008) 116001 [arXiv: 0712 . 1882]; C.-W. Chiang, E. Senaha, Updated analysis of two-body charmed B meson decays, Phys. Rev. D 75 (2007) 074021 [arXiv:hep-ph/0702007]

[7] M. Gronau et al., Using untagged $B^{0} \rightarrow D K_{S}$ to determine $\gamma$, Phys. Rev. D 69 (2004) 113003 [arXiv:hep-ph/0402055]

[8] R. Fleischer, New, efficient and clean strategies to explore CP violation through neutral B decays, Phys. Lett. B 562 (2003) 234 [arXiv: hep-ph/0301255]

[9] LHCb collaboration, Search for the decay $B_{s}^{0} \rightarrow \bar{D}^{0} f_{0}(980)$, JHEP $\mathbf{0 8}$ (2015) 005 [arXiv:1505.01654]

[10] R. Fleischer, R. Knegjens, G. Ricciardi, Anatomy of $B_{s, d}^{0} \rightarrow J / \psi f_{0}(980)$, Eur. Phys. J. C 71 (2011) 1832 [arXiv:1109.1112]; S. Stone, L. Zhang, Use of $B \rightarrow J / \psi f_{0}$ decays to discern the $q \bar{q}$ or tetraquark nature of scalar mesons, Phys. Rev. Lett. 111 (2013) 062001 [arXiv: 1305.6554 ] 
[11] Belle collaboration, Study of $\bar{B}^{0} \rightarrow D^{0} \pi^{+} \pi^{-}$decays, Phys. Rev. D 76 (2007) 012006 [arXiv:hep-ex/0611054]

[12] LHCb collaboration, Dalitz plot analysis of $B^{0} \rightarrow \bar{D}^{0} \pi^{+} \pi^{-}$decays, Phys. Rev. D 92 (2015) 032002 [arXiv:1505.01710]

[13] M. Gronau, D. London, How to determine all the angles of the unitarity triangle from $B^{0} \rightarrow D K_{S}^{0}$ and $B_{s}^{0} \rightarrow$ D $\phi$, Phys. Lett. B 253 (1991) 483; M. Gronau et al., Enhanced effects on extracting $\gamma$ from untagged $B^{0}$ and $B_{s}^{0}$ decays, Phys. Lett. B 649 (2007) 61 [arXiv: hep-ph/ 0702011 ]; S. Nandi, D. London, $B_{s}\left(\bar{B}_{S}\right) \rightarrow D_{C P}^{0} K \bar{K}$ : detecting and discriminating new physics in $B_{s}-\bar{B}_{s}$ mixing, Phys. Rev. $D$ 85 (2012) 114015 [arXiv: 1108 . 5769]; W. Wang, Determining CP-violation angle $\gamma$ with $B$ decays into a scalar/tensor meson, Phys. Rev. D 85 (2012) 051301 [arXiv: 1110 . 5194]

[14] LHCb collaboration, First observation of the rare $B^{+} \rightarrow D^{+} K^{+} \pi^{-}$decay, Phys. Rev. D 93 (2016) 051101(R) [arXiv:1512.02494]

[15] LHCb collaboration, First observation and amplitude analysis of the $B^{-} \rightarrow D^{+} K^{-} \pi^{-}$decay, Phys. Rev. D 91 (2015) 092002 [arXiv: 1503 .02995]

[16] N. Sinha, Determining $\gamma$ using $B \rightarrow D^{* *} K$, Phys. Rev. D 70 (2004) 097501 [arXiv:hep-ph/0405061]

[17] J. Charles et al., $B_{d}^{0}(t) \rightarrow D P P$ time-dependent Dalitz plots, $C P$-violating angles $2 \beta, 2 \beta+\gamma$, and discrete ambiguities, Phys. Lett. B 425 (1998) 375 [arXiv: hep-ph/9801363]; T. Latham, T. Gershon, A method of measuring $\cos (2 \beta)$ using a time-dependent Dalitz plot analysis of $B^{0} \rightarrow D_{C P} \pi^{+} \pi^{-}$, J. Phys. G 36 (2009) 025006 [arXiv: 0809 . 0872]; BABAR collaboration, Measurement of the time-dependent CP asymmetry in $B^{0} \rightarrow D_{C P}^{(*)} h^{0}$ decays, Phys. Rev. Lett. 99 (2007) 081801 [arXiv: hep-ex/0 703019]; BABAR collaboration, Measurement of $\cos (2 \beta)$ in $B^{0} \rightarrow D^{(*)} h^{0}$ decays with a time-dependent Dalitz plot analysis of $D \rightarrow K_{S}^{0} \pi^{+} \pi^{-}$, Phys. Rev. Lett. 99 (2007) 231802 [arXiv:0708.1544]; Belle collaboration, Measurement of the quark mixing parameter $\cos \left(2 \phi_{1}\right)$ using time-dependent Dalitz analysis of $\bar{B}^{0} \rightarrow D\left[K_{S}^{0} \pi^{+} \pi^{-}\right] h^{0}$, Phys. Rev. Lett. 97 (2006) 081801 [arXiv: hep-ex/0605023]

[18] Y. Grossman, M. P. Worah, CP asymmetries in B decays with new physics in decay amplitudes, Phys. Lett. B 395 (1997) 241 [arXiv: hep-ph/9612269]; R. Fleischer, CP violation and the role of electroweak penguins in nonleptonic B decays, Int. J. Mod. Phys. A 12 (1997) 2459 [arXiv: hep-ph/9612446]; D. London, A. Soni, Measuring the $C P$ angle $\beta$ in hadronic $b \rightarrow s$ penguin decays, Phys. Lett. B 407 (1997) 61 [arXiv: hep-ph/ 9704277 ]; M. Ciuchini et al., CP violating B decays in the Standard Model and Supersymmetry, Phys. Rev. Lett. 79 (1997) 978 [arXiv:hep-ph/9704274]

[19] LHCb collaboration, Amplitude analysis of $B^{0} \rightarrow \bar{D}^{0} K^{+} \pi^{-}$decays, Phys. Rev. D 92 (2015) 012012 [arXiv: 1505.01505]

[20] T. Gershon, On the measurement of the unitarity triangle angle $\gamma$ from $B^{0} \rightarrow D K^{* 0}$ decays, Phys. Rev. D 79 (2009) 051301(R) [arXiv: 0810 . 2706]; T. Gershon, M. Williams, Prospects for the measurement of the unitarity triangle angle $\gamma$ from $B^{0} \rightarrow D K^{+} \pi^{-}$decays, Phys. Rev. D 80 (2009) 092002 [arXiv:0909.1495]

[21] LHCb collaboration, Constraints on the unitarity triangle angle $\gamma$ from Dalitz plot analysis of $B^{0} \rightarrow D K^{+} \pi^{-}$decays, Submitted to Phys. Rev. Lett. [arXiv:1602.03455]

[22] S.-F. Cheung, these proceedings; LHCb collaboration, Measurement of the CKM angle $\gamma$ from a combination of $B \rightarrow D K$ analyses, LHCb-CONF-2016-001 (2016) 